\title{
CURRENT MINOR PLANET PROBLEMS
}

\author{
By PAUL HERGET
}

The problems of the Minor Planet Center are: (I) the tremendous number of planets. This is introducing a new "selection rule" in the ones which are being followed, e.g., the practice on the Lick 20-inch. (2) Interruptions by the war now require many differential corrections to make more reliable predictions for recovery. (3) Numerous misidentifications of isolated observations. It is absolutely essential that confirming observations be made a month later in all cases of insecure objects. (4) The limited observing equipment devoted to this work. (5) Changes in policy due to the use of large scale computing equipment for perturbations. Computation should absorb more of the role which observations have played in keeping track of the planets. Several large programs of computation of perturbations using different methods, have now been completed at the Cincinnati Observatory. The identifications of unnumbered planets, and the recoveries of numbered ones by searching, especially at the Link Observatory, are very helpful. More assistance is needed in making differential corrections.

Cincinnati Observatory, Cincinnati, Ohio

\section{ABSTRACTS*}

Bidelman, William P. The absolute magnitude of $\mathrm{R}$ Puppis.

Three very luminous stars appear to be associated with the distant B star galactic cluster, NGC 2439; the brightest of these is the Go la star R Puppis. The others are an early B supergiant star spectroscopically intermediate between $\zeta$ Per and $\epsilon \mathrm{CMa}, \mathrm{CPD}-3 \mathrm{I}^{\circ} \mathrm{I} 785$, and $\mathrm{a}$ red supergiant of type $\mathrm{M} 2 \mathrm{Ia}-\mathrm{Ib}, \mathrm{CPD}-3 \mathrm{I}^{\circ} \mathrm{I} 790$. The estimated spectroscopic visual absolute magnitude of the B-type star is about -5.2 , which implies a visual absolute magnitude of -7.2 for $R$ Puppis and of -5.6 for the M-type supergiant star. Though the main sequence of the cluster has not been observed, at least one fainter cluster member of intermediate luminosity at class $\mathrm{B}_{2}$ is consistent with these values. The three supergiant stars are not greatly different in bolometric magnitude. NGC 2439 is the only known case of the simultaneous presence of a B-, a G-, and an M-type supergiant star in a cluster containing predominantly stars of early type.

Yerkes Observatory, Williams Bay, Wis.

Blitzstein, William. The eclipsing variable $\mathrm{XZ}$ Andromedae.

Observations were made of the light variation of $\mathrm{XZ}$ Andromedae with the pulse-counting photometer of the Cook Observatory of the University of Pennsylvania. Times of minimum were observed and new light elements for pre- diction in the immediate future were derived. The light between eclipses showed the ellipticity effect and no reflection effect. A small oscillation whose amplitude is about I per cent of the combined light was detected. This is best represented by an out-of-phase fifth harmonic of the period. The primary minimum is best represented by an occultation with $k=0.965$ and $x=0.4$. The corresponding computed secondary eclipse, assuming a circular orbit, did not fit the observations well. Minor adjustments of the rectification and depths did not give a satisfactory representation. An assumption of an eccentric orbit with $e \cos \omega=0$ and $e \sin \omega=0$. I I gave a good fit to the observations at secondary. The system was unstable for a mass ratio greater than $\mathbf{\text { I.6 }}$ using as a criterion the Jacobian limiting surface and the equatorial minor axis of the less massive star. The mass ratio by the mass-luminosity law was 2.9 .

Flower Observatory, Upper Darby, Pa.

\section{Brown, Archibald. Color-magnitude array for} stars in the globular cluster M I5.

The globular cluster M I5 $\left(\alpha=2 \mathrm{I}^{\mathrm{h}} 28^{\mathrm{m}}\right.$, $\left.\delta=+\mathrm{II}^{\circ} 57^{\prime}\right)$ is one which is moderately rich in variables and shows an appreciable concentration toward the centre. The paper gives colors and magnitudes for stars in this cluster, using

* Of papers presented at the Eighty-third Meeting of the American Astronomical Society, Bloomington, Ind., June I 8-2 I, I950. 